\title{
Hidden spin-3/2 field in the standard model
}

\author{
Durmuş Demir $^{1, \mathrm{a}}$, Canan Karahan ${ }^{1, \mathrm{~b}}$, Beste Korutlu ${ }^{2, \mathrm{c}}$, Ozan Sargın ${ }^{1, \mathrm{~d}}$ \\ ${ }^{1}$ Department of Physics, İzmir Institute of Technology, 35430 Urla, Izmir, Turkey \\ 2 TÜBİTAK National Metrology Institute, 41470 Gebze, Kocaeli, Turkey
}

Received: 13 February 2017 / Accepted: 25 August 2017 / Published online: 8 September 2017

(C) The Author(s) 2017. This article is an open access publication

\begin{abstract}
Here we show that a massive spin-3/2 field can hide in the SM spectrum in a way revealing itself only virtually. We study collider signatures and loop effects of this field, and determine its role in Higgs inflation and its potential as dark matter. We show that this spin-3/2 field has a rich linear collider phenomenology and motivates consideration of a neutrino-Higgs collider. We also show that the study of Higgs inflation, dark matter and dark energy can reveal more about the neutrino and dark sector.
\end{abstract}

\section{Introduction}

The standard model (SM) of strong and electroweak interactions, spectrally completed by the discovery of its Higgs boson at the LHC [1], seems to be the model of physics at Fermi energies. It does so because various experiments have revealed so far no new particles beyond the SM spectrum. There is, however, at least the dark matter (DM), which requires new particles beyond the SM. Physically, therefore, we must use every opportunity to understand where those new particles can hide, if there are any.

In the present work we study a massive spin-3/2 field hidden in the SM spectrum. This higher-spin field, described by the Rarita-Schwinger equations [2-4], has to obey certain constraints to have correct degrees of freedom when it is on the physical shell. At the renormalizable level, it can couple to the SM matter via only the neutrino portal (the composite SM singlet formed by the lepton doublet and the Higgs field). This interaction is such that it vanishes when the spin$3 / 2$ field is on shell. In Sect. 2 below we give the model and basic constraints on the spin-3/2 field.

This work is supported in part by the TUBITAK Grant 115F212.

\footnotetext{
a e-mail: demir@physics.iztech.edu.tr

be-mail: cananduzturk@iyte.edu.tr

c e-mail: beste.korutlu@tubitak.gov.tr

de-mail: ozansargin@iyte.edu.tr
}

In Sect. 3 we study collider signatures of the spin-3/2 field. We study there $v_{L} h \rightarrow v_{L} h$ and $e^{-} e^{+} \rightarrow W^{+} W^{-}$ scatterings in detail. We give analytical computations and numerical predictions. We propose there a neutrino-Higgs collider and emphasize the importance of the linear collider in probing the spin-3/2 field.

In Sect. 4 we turn to loop effects of the spin-3/2 field. We find that the spin-3/2 field adds logarithmic and quartic UVsensitivities atop the logarithmic and quadratic ones in the SM. We convert power-law UV-dependent terms into curvature terms as a result of the incorporation of gravity into the SM. Here we use the results of [5-7], which show that gravity can be incorporated into the SM properly and naturally (i) if the requisite curved geometry is structured by interpreting the UV cutoff as a constant value assigned to the spacetime curvature, and (ii) if the SM is extended by a secluded new physics (NP) that does not have to interact with the SM. This mechanism eliminates the big hierarchy problem by metamorphosing the quadratic UV part of the Higgs boson mass turns into Higgs-curvature coupling.

In Sect. 5 we discuss the possibility of Higgs inflation via the large Higgs non-minimal coupling induced by the spin$3 / 2$ field. We find that Higgs inflation is possible in a wide range of parameters provided that the secluded NP sector is crowded enough.

In Sect. 6 we discuss the DM. We show therein that the spin-3/2 field is a viable DM candidate. We also show that the singlet fields in the NP can form a non-interacting DM component.

In Sect. 7 we conclude. There, we give a brief list of problems that can be studied as furthering of the material presented in this work.

\section{A light spin-3/2 field}

Introduced for the first time by Rarita and Schwinger [2], $\psi_{\mu}$ propagates with 
$S^{\alpha \beta}(p)=\frac{i}{\not p-M} \Pi^{\alpha \beta}(p)$,

carrying one spin-3/2 and two spin- $1 / 2$ components through the projector $[3,4]$,

$\Pi^{\alpha \beta}=-\eta^{\alpha \beta}+\frac{\gamma^{\alpha} \gamma^{\beta}}{3}+\frac{\left(\gamma^{\alpha} p^{\beta}-\gamma^{\beta} p^{\alpha}\right)}{3 M}+\frac{2 p^{\alpha} p^{\beta}}{3 M^{2}}$,

which exhibits both spinor and vector characteristics. It is necessary to impose $[3,4]$

$\left.p^{\mu} \psi_{\mu}(p)\right\rfloor_{p^{2}=M^{2}}=0$

and

$\left.\gamma^{\mu} \psi_{\mu}(p)\right\rfloor_{p^{2}=M^{2}}=0$,

to eliminate the two spin- $1 / 2$ components to make $\psi_{\mu}$ satisfy the Dirac equation

$$
(\not p-M) \psi_{\mu}=0
$$

as expected of an on-shell fermion. The constraints (3) and (4) imply that $p^{\mu} \psi_{\mu}(p)$ and $\gamma^{\mu} \psi_{\mu}(p)$ both vanish on the physical shell $p^{2}=M^{2}$. The latter is illustrated in Fig. 1 taking $\psi_{\mu}$ on shell.

Characteristic of singlet fermions, the $\psi_{\mu}$, at the renormalizable level, makes contact with the SM via

$\mathcal{L}_{3 / 2}^{(i n t)}=c_{3 / 2}^{i} \overline{L^{i}} H \gamma^{\mu} \psi_{\mu}+$ h.c.

in which

$L^{i}=\left(\begin{array}{c}v_{\ell L} \\ \ell_{L}\end{array}\right)_{i}$

is the lepton doublet $(i=1,2,3)$ and

$H=\frac{1}{\sqrt{2}}\left(\begin{array}{c}v+h+i \varphi^{0} \\ \sqrt{2} \varphi^{-}\end{array}\right)$

is the Higgs doublet with vacuum expectation value $v \approx$ $246 \mathrm{GeV}$, Higgs boson $h$, and Goldstone bosons $\varphi^{-}, \varphi^{0}$ and $\varphi^{+}$(forming the longitudinal components of $W^{-}, Z$ and $W^{+}$ bosons, respectively).

In general, neutrinos are sensitive probes of singlet fermions. They can get masses through, for instance, the Yukawa interaction (6), which leads to the Majorana mass matrix

$\left(m_{v}\right)_{3 / 2}^{i j} \propto c_{3 / 2}^{i} \frac{v^{2}}{M} c_{3 / 2}^{\star j}$

after integrating out $\psi_{\mu}$. This mass matrix, however, cannot lead to the experimentally known neutrino mixings [8-10]. This means that flavor structures necessitate additional singlet fermions. Of such a type are the right-handed neutrinos $v_{R}^{k}$ of mass $M_{k}(k=1,2,3, \ldots)$, which interact with the SM through

$\mathcal{L}_{R}^{(i n t)}=c_{R}^{i k} \bar{L}^{i} H v_{R}^{k}+$ h.c.

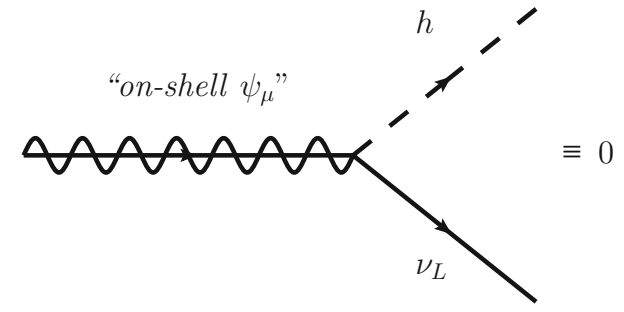

Fig. $1 \psi_{\mu}-h-v_{L}$ coupling with vertex factor $i c_{3 / 2} \gamma^{\mu}$. Scatterings in which $\psi_{\mu}$ is on shell must all be forbidden since $c_{3 / 2} \gamma^{\mu} \psi_{\mu}$ vanishes on mass shell by the constraint (4). This ensures stability of $\psi_{\mu}$ against decays and all sort of co-annihilations

to generate the neutrino Majorana masses

$\left(m_{v}\right)_{R}^{i j} \propto c_{R}^{i k} \frac{v^{2}}{M_{k}} c_{R}^{\star k j}$

of more general flavor structure. This mass matrix must have enough degrees of freedom to fit to the data [8-10].

Here we make a pivotal assumption. We assume that $\psi_{\mu}$ and $v_{R}^{k}$ can weigh as low as a $\mathrm{TeV}$, and that $c_{3 / 2}^{i}$ and some of $c_{R}^{i k}$ can be $\mathcal{O}(1)$. We, however, require that contributions to neutrino masses from $\psi_{\mu}$ and $v_{R}$ add up to reproduce the experimental result

$\left(m_{v}\right)_{3 / 2}^{i j}+\left(m_{v}\right)_{R}^{i j} \approx\left(m_{v}\right)_{\exp }^{i j}$

via cancellations among different terms. We therefore take

$c_{3 / 2} \lesssim \mathcal{O}(1), \quad M \gtrsim \mathrm{TeV}$

and investigate the physics of $\psi_{\mu}$. This cancellation requirement does not have to cause any excessive fine-tuning simply because $\psi_{\mu}$ and $v_{R}^{k}$ can have appropriate symmetries that correlate their couplings. One possible symmetry would be rotation of $\gamma^{\mu} \psi_{\mu}$ and $v_{R}^{k}$ into each other. We defer study of possible symmetries to another work, which is in progress [11]. The right-handed sector, which can involve many $v_{R}^{k}$ fields, is interesting by itself but hereon we focus on $\psi_{\mu}$ and take, for simplicity, $c_{3 / 2}^{i}$ real and family-universal $\left(c_{3 / 2}^{i}=c_{3 / 2}\right.$ for $\forall i)$.

\section{Spin-3/2 field at colliders}

It is only when it is off-shell that $\psi_{\mu}$ can reveal itself through the interaction (6). This means that its effects are restricted to modifications in scattering rates of the SM particles. To this end, as follows from (6), it participates in

1. $v_{L} h \rightarrow v_{L} h$ (and also $v_{L} v_{L} \rightarrow h h$ ),

2. $e^{+} e^{-} \rightarrow W_{L}^{+} W_{L}^{-}$(and also $v_{L} v_{L} \rightarrow Z_{L} Z_{L}$ ),

at the tree level. They are analyzed below in detail. 


\section{$3.1 v_{L} h \rightarrow v_{L} h$ Scattering}

Shown in Fig. 2 are the two box diagrams which enable $v_{L} h \rightarrow v_{L} h$ scattering in the SM. Added to this loopsuppressed SM piece is the $\psi_{\mu}$ piece depicted in Fig. 3. The two contributions add up to give the cross section

$$
\frac{\mathrm{d} \sigma\left(v_{L} h \rightarrow v_{L} h\right)}{\mathrm{d} t}=\frac{1}{16 \pi} \frac{\mathcal{T}_{v h}(s, t)}{\left(s-m_{h}^{2}\right)^{2}},
$$

in which the squared matrix element

$$
\begin{aligned}
\mathcal{T}_{v h}(s, t)= & 9\left(\frac{c_{3 / 2}}{3 M}\right)^{4}\left(\left(s-m_{h}^{2}\right)^{2}+s t\right) \\
& -16\left(\frac{c_{3 / 2}}{3 M}\right)^{2}\left(2\left(s-m_{h}^{2}\right)^{2}+\left(2 s-m_{h}^{2}\right) t\right) \\
& \times \mathbb{L}+2\left(s-m_{h}^{2}\right)\left(s+t-m_{h}^{2}\right) \mathbb{L}^{2}
\end{aligned}
$$

involves the loop factor

$\mathbb{L}=\frac{\left(g_{W}^{2}+g_{Y}^{2}\right)^{2} M_{Z}^{2} m_{h}^{2} I\left(M_{Z}\right)}{192 \pi^{2}}+\frac{g_{W}^{4} M_{W}^{2} m_{h}^{2} I\left(M_{W}\right)}{96 \pi^{2}}$

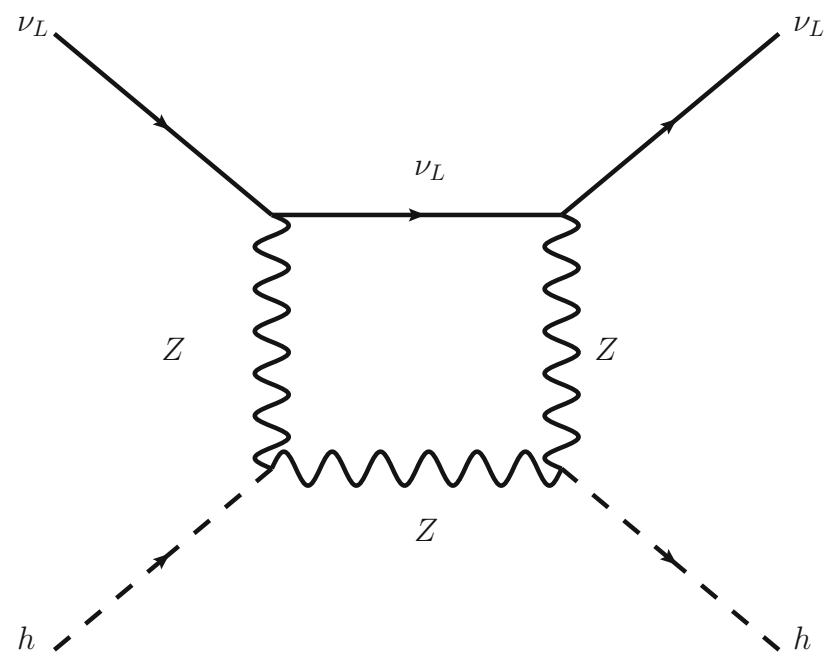

Fig. 2 The $v-Z$ box mediating the $v_{L} h \rightarrow v_{L} h$ scattering in the SM. The $e-W$ box is not shown

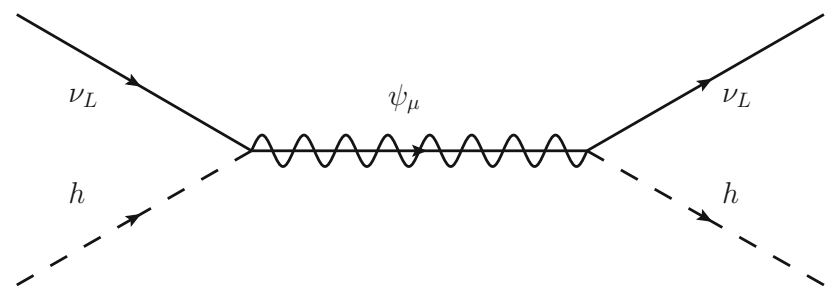

Fig. $3 v_{L} h \rightarrow v_{L} h$ scattering with $\psi_{\mu}$ mediation. No resonance can occur at $\sqrt{s}=M$ because $\psi_{D M}$ cannot come to mass shell in which $g_{W}\left(g_{Y}\right)$ is the isospin (hypercharge) gauge coupling, and

$$
\begin{aligned}
I(\mu)= & \int_{0}^{1} \mathrm{~d} x \int_{0}^{1-x} \mathrm{~d} y \int_{0}^{1-x-y} \\
& \times \mathrm{d} z\left(\left(s-m_{h}^{2}\right)(x+y+z-1) y\right. \\
& \left.-t x z+m_{h}^{2} y(y-1)+\mu^{2}(x+y+z)\right)^{-2}
\end{aligned}
$$

is the box function. In Fig. 4, we plot the total cross section $\sigma\left(v_{L} h \rightarrow v_{L} h\right)$ as a function of the neutrino-Higgs centerof-mass energy for different $M$ values. The first important thing about the plot is that there is no resonance formation around $\sqrt{s}=M$. This confirms the fact that $\psi_{\mu}$, under the constraint (4), cannot come to physical shell with the couplings in (6). In consequence, the main search strategy for $\psi_{\mu}$ is to look for deviations from the SM rates rather than resonance shapes. The second important thing about the plot is that, in general, as revealed by (19), the larger $M$, the smaller the $\psi_{\mu}$ contribution. The cross section starts around $10^{-7} \mathrm{pb}$, and falls rapidly with $\sqrt{s}$. (The SM piece, as a loop effect, is too tiny to be observable: $\sigma\left(v_{L} h \rightarrow v_{L} h\right) \lesssim 10^{-17} \mathrm{pb}$.) It is necessary to have some $10^{4} / \mathrm{fb}$ integrated luminosity (100 times the target luminosity at the LHC) to observe a few events in a year. This means that $v_{L} v_{L} \rightarrow h h$ scattering can probe $\psi_{\mu}$ only at high luminosity but with a completely new scattering scheme.

Figure 4 shows that neutrino-Higgs scattering can be a promising channel to probe $\psi_{\mu}$ (at high-luminosity, highenergy machines). The requisite experimental setup would involve crossing of Higgs factories with accelerator neutrinos. The setup, schematically depicted in Fig. 5, can be viewed as incorporating future Higgs (CEPC [12], FCC-ee $[13,14]$ and ILC [15-17]) and neutrino [18-20] factories. If

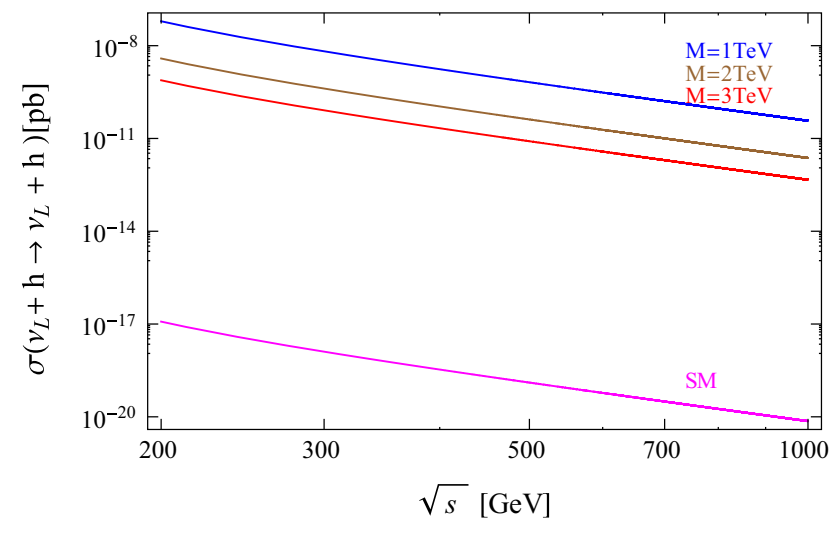

Fig. 4 The total cross section for $v_{L} h \rightarrow v_{L} h$ scattering as a function of the neutrino-Higgs center-of-mass energy $\sqrt{s}$ for $M=1,2$ and $3 \mathrm{TeV}$ at $c_{3 / 2}=1$. Cases with $c_{3 / 2} \neq 1$ can be reached via the rescaling $M \rightarrow M / c_{3 / 2}$ 


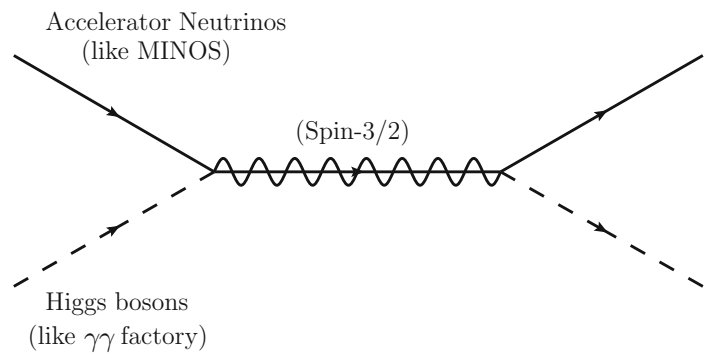

Fig. 5 Possible neutrino-Higgs collider to probe $\psi_{\mu}$

ever realized, it could be a rather clean experiment with negligible SM background. This hypothetical "neutrino-Higgs collider", depicted in Fig. 5, must have, as suggested by Fig. 4 , some $10^{4} / \mathrm{fb}$ integrated luminosity to be able to probe a $\mathrm{TeV}$-scale $\psi_{\mu}$. In general, the need to have high luminosities is a disadvantage of this channel. (A feasibility study, technical design and possible realization of a "neutrino-Higgs collider" fall outside the scope of the present work.)

\section{$3.2 e^{+} e^{-} \rightarrow W_{L}^{+} W_{L}^{-}$Scattering}

It is clear that $\psi_{\mu}$ directly couples to the Goldstone bosons $\varphi^{+,-, 0}$ via (6). The Goldstones, though eaten up by the $W$ and $Z$ bosons in acquiring their masses, reveal themselves at high energies. In fact, the Goldstone equivalence theorem [21-23] states that scatterings at energy $E$ involving longitudinal $W_{L}^{ \pm}$bosons are equal to scatterings that involve $\varphi^{ \pm}$ up to terms $\mathcal{O}\left(M_{W}^{2} / E^{2}\right)$. This theorem, with similar equivalence for the longitudinal $Z$ boson, provides a different way of probing $\psi_{\mu}$. In this regard, depicted in Fig. 6 is $\psi_{\mu}$ contribution to $e^{+} e^{-} \rightarrow W_{L}^{+} W_{L}^{-}$scattering in light of the Goldstone equivalence. The $\mathrm{SM}$ amplitude is given in [21-23]. The total differential cross section

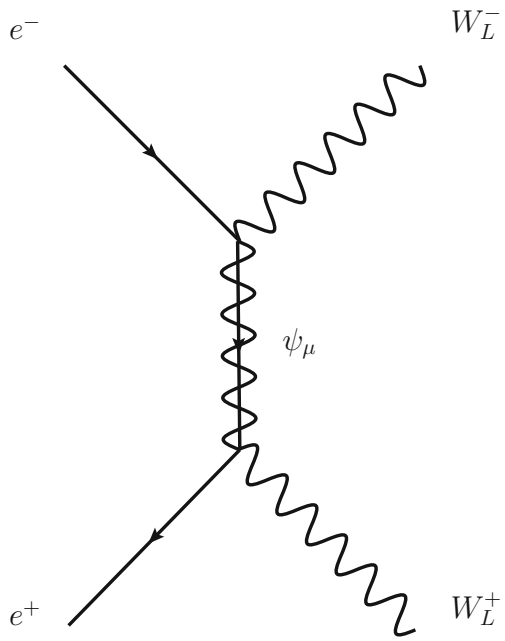

Fig. 6 The Feynman diagram for $e^{+} e^{-} \rightarrow W_{L}^{+} W_{L}^{-}$scattering. The $v_{L} v_{L} \rightarrow Z_{L} Z_{L}$ scattering has the same topology $\frac{\mathrm{d} \sigma\left(e^{+} e^{-} \rightarrow W_{L}^{+} W_{L}^{-}\right)}{\mathrm{d} t}=\frac{1}{16 \pi s^{2}} \mathcal{T}_{W_{L} W_{L}}(s, t)$

involves the squared matrix element

$$
\begin{aligned}
\mathcal{T}_{W_{L} W_{L}}(s, t)= & \left(\frac{g_{W}^{2}}{s-M_{Z}^{2}}\left(-1+\frac{M_{Z}^{2}}{4 M_{W}^{2}}+\frac{M_{Z}^{2}-M_{W}^{2}}{s}\right)\right. \\
& \left.+\frac{g_{W}^{2}}{s-4 M_{Z}^{2}}\left(1+\frac{M_{W}^{2}}{t}\right)+\frac{c_{3 / 2}^{2}}{3 M^{2}}\right)^{2} \\
& \times\left(-2 s M_{W}^{2}-2\left(t-M_{W}^{2}\right)^{2}\right) \\
& +\frac{c_{3 / 2}^{4} s}{18 M^{2}}\left(4+\frac{t}{t-M^{2}}\right)^{2} .
\end{aligned}
$$

Plotted in Fig. 7 is $\sigma\left(e^{+} e^{-} \rightarrow W_{L}^{+} W_{L}^{-}\right)$as a function of the $e^{+} e^{-}$center-of-mass energy for different values of $M$. The cross section, which falls with $\sqrt{s}$ without exhibiting a resonance shape, is seen to be large enough to be measurable at the ILC [15-17]. In general, the larger $M$, the smaller the cross section but even $1 / f b$ luminosity is sufficient for probing $\psi_{\mu}$ for a wide range of mass values.

Collider searches for $\psi_{\mu}$, as illustrated by $v_{L} h \rightarrow v_{L} h$ and $e^{-} e^{+} \rightarrow W^{+} W^{-}$scatterings, can access spin-3/2 fields of several $\mathrm{TeV}$ mass. For instance, the ILC, depending on its precision, can confirm or exclude a $\psi_{\mu}$ of even $5 \mathrm{TeV}$ mass with an integrated luminosity around $1 / f b$. Depending on the possibility and feasibility of a neutrino-neutrino collider (mainly accelerator neutrinos), it may be possible to study also $v_{L} v_{L} \rightarrow h h$ and $v_{L} v_{L} \rightarrow Z_{L} Z_{L}$ scatterings, which are expected to have similar sensitivities to $M$.

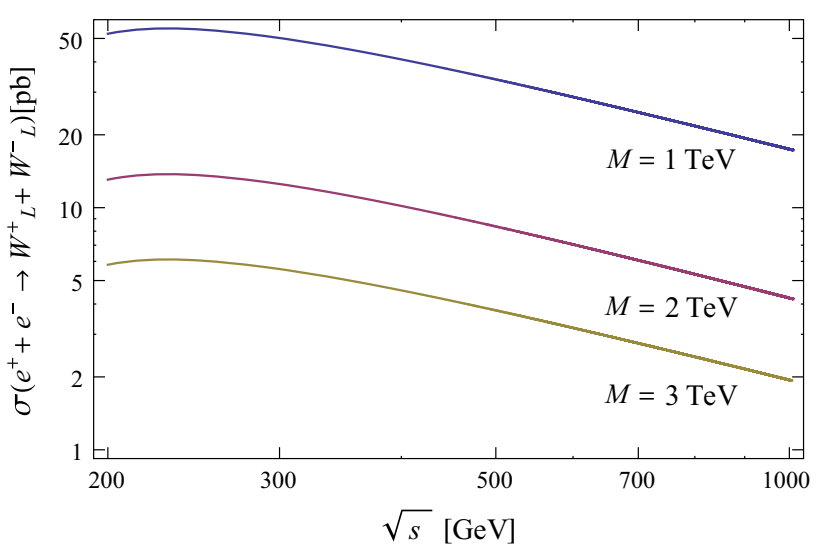

Fig. 7 The total cross section for $e^{-} e^{+} \rightarrow W^{+} W^{-}$scattering as a function of the electron-positron center-of-mass energy $\sqrt{s}$ for $M=$ 1,2 and $3 \mathrm{TeV}$ at $c_{3 / 2}=1$. Cases with $c_{3 / 2} \neq 1$ can be reached via the rescaling $M \rightarrow M / c_{3 / 2}$ 


\section{Spin-3/2 field in loops}

As an inherently off-shell field, $\psi_{\mu}$ is expected to reveal itself mainly in loops. Its only possible loop effect would be generation of neutrino masses but chirality forbids it. Despite the couplings in (6), therefore, neutrino masses do not get any contribution from the $\psi_{\mu}-h$ loop.

One other loop effect of $\psi_{\mu}$ would be radiative corrections to the Higgs boson mass. This is not forbidden by any symmetry. The relevant Feynman diagram is depicted in Fig. 8. It adds to the Higgs boson squared-mass a logarithmic piece,

$$
\left(\delta m_{h}^{2}\right)_{\log }=\frac{c_{3 / 2}^{2}}{12 \pi^{2}} M^{2} \log G_{F} M^{2},
$$

relative to the logarithmic piece $\log G_{F} \Lambda^{2}$ in the SM and a quartic piece

$$
\left(\delta m_{h}^{2}\right)_{4}=\frac{c_{3 / 2}^{2}}{48 \pi^{2}} \frac{\Lambda^{4}}{M^{2}},
$$

which have the potential to override the experimental result [1] depending on how large the UV cutoff $\Lambda$ is compared to the Fermi scale $G_{F}^{-1 / 2}=293 \mathrm{GeV}$.

The logarithmic contribution in (20), which originates from the $\eta^{\alpha \beta}$ part of (2), gives rise to the little hierarchy problem in that the larger $M$, the stronger the destabilization of the SM Higgs sector. Leaving aside the possibility of cancellations with similar contributions from the right-handed neutrinos $v_{R}^{k}$ in (10), the little hierarchy problem can be prevented if $M$ (more precisely $M / c_{3 / 2}$ ) lies in the TeV domain.

The quartic contribution in (21), which originates from the longitudinal $p^{\alpha} p^{\beta}$ term in (2), gives cause to the notorious big hierarchy problem in that the larger $\Lambda$ is, the larger the destabilization of the SM Higgs sector. This power-law UV sensitivity exists already in the SM

$$
\left(\delta m_{h}^{2}\right)_{2}=\frac{3 \Lambda^{2}}{16 \pi^{2}|\langle H\rangle|^{2}}\left(m_{h}^{2}+2 M_{W}^{2}+M_{Z}^{2}-4 m_{t}^{2}\right)
$$

at the quadratic level [24] and violates the LHC bounds unless $\Lambda \lesssim 550 \mathrm{GeV}$. This bound obviously contradicts with the LHC experiments since the latter continue to confirm the

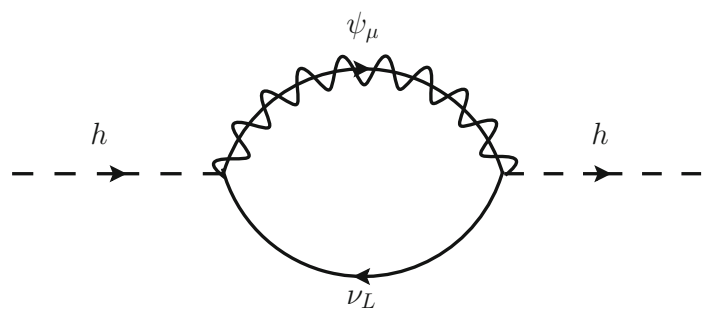

Fig. 8 The $\psi_{\mu}-v_{L}$ loop that generates the logarithmic correction in (20) and the quartic correction in (21)
$\mathrm{SM}$ at multi $\mathrm{TeV}$ energies. This experimental fact makes it obligatory to find a natural UV completion to the SM.

One possibility is to require $\left(\delta m_{h}^{2}\right)_{4}$ to cancel out $\left(\delta m_{h}^{2}\right)_{2}$. This requirement involves a severe fine-tuning (as with a scalar field [31-34], Stueckelberg vector [35] and spacetime curvature $[36,37])$ and cannot form a viable stabilization mechanism.

Another possibility would be to switch, for instance, to a dimensional regularization scheme, wherein the quartic and quadratic UV-dependencies are known to disappear. This, however, is not a solution. The reason is that the SM, as a quantum field theory of the strong and electroweak interactions, needs gravity to be incorporated as the fourth known force. And the fundamental scale of gravity, $M_{P l}$, inevitably sets an non-eliminable physical UV cutoff (rendering $\Lambda$ physical). This cutoff forces quantum field theories to exist in between physical UV and IR scales. The SM plus $\psi_{\mu}$ (plus right-handed neutrinos), for instance, ranges from $G_{F}^{-1 / 2}$ at the IR up to $\Lambda$ at the UV such that both scales are physical (not to be confused with the formal momentum cutoffs employed in the cutoff regularization).

To stabilize the SM, it is necessary to metamorphose the destabilizing UV effects. This necessitates a physical agent. The most obvious candidate is gravity. That is to say, the UV-naturalness problems can be a clue to how quantized matter must gravitate. Indeed, quantized matter in classical curved geometry suffers from inconsistencies. The situation can be improved by considering long-wavelength matter by integrating out high-frequency modes. This means that the theory to be carried into curved geometry for incorporating gravity is not the full action but the effective action (see the discussions in [5-7]). Thus, starting with the SM effective action in flat spacetime with well-known logarithmic, quartic and quadratic UV-sensitivities, gravity can be incorporated in a way that ensures UV-naturalness. More precisely, gravity gets incorporated properly and naturally (i) if the requisite curved geometry is structured by interpreting $\Lambda^{2}$ as a constant value assigned to the spacetime curvature, and (ii) if the SM is extended by new physics (NP) that does not have to interact with the SM. The $\psi_{\mu}$ can well be an NP field. Incorporating gravity by identifying $\Lambda^{2} g_{\mu \nu}$ with the Ricci curvature $R_{\mu \nu}(g)$, fundamental scale of gravity gets generated as

$M_{P l}^{2} \approx \frac{\left(n_{b}-n_{f}\right)}{2(8 \pi)^{2}} \Lambda^{2}$

where $n_{b}\left(n_{f}\right)$ are the total number of bosons (fermions) in the SM plus the NP. The $\psi_{\mu}$ increases $n_{f}$ by 4 , right-handed neutrinos by 2 . There are various other fields in the NP, which contribute to $n_{b}$ and $n_{f}$ to ensure $\Lambda \lesssim M_{P l}$. Excepting $\psi_{\mu}$, they do not need to interact with the SM fields. Induction of $M_{P l}$ ensures that the quadratic UV-contributions to vacuum energy are canalized not to the cosmological constant but to 
the gravitational constant (see $[38,39]$, arriving at this result in a different context). This suppresses the cosmological constant down to the neutrino mass scale.

The quartic UV-contributions in (21) and the quadratic contributions in (22) (suppressing contributions from the right-handed neutrinos $v_{R}^{k}$ ) change their roles with the inclusion of gravity. Indeed, corrections to the Higgs mass term $\left[\left(\delta m_{h}^{2}\right)_{4}+\left(\delta m_{h}^{2}\right)_{2}\right] H^{\dagger} H$ turn into

$$
\left[\frac{3\left(m_{h}^{2}+2 M_{W}^{2}+M_{Z}^{2}-4 m_{t}^{2}\right)}{(8 \pi)^{2}|\langle H\rangle|^{2}}+\frac{c_{3 / 2}^{2}}{12\left(n_{b}-n_{f}\right)} \frac{M_{P l}^{2}}{M^{2}}\right] R H^{\dagger} H,
$$

which is nothing but the direct coupling of the Higgs field to the scalar curvature $R$. This Higgs-curvature coupling is perfectly natural; it has no potential to de-stabilize the Higgs sector. Incorporation of gravity as in [5-7] leads, therefore, to UV-naturalization of the SM with a nontrivial NP sector containing $\psi_{\mu}$ as its interacting member.

\section{Spin-3/2 field as enabler of higgs inflation}

The non-minimal Higgs-curvature coupling in (24) reminds one at once of the possibility of Higgs inflation. Indeed, the Higgs field has been shown in $[40,41]$ to lead to correct inflationary expansion provided that

$\frac{c_{3 / 2}^{2}}{12\left(n_{b}-n_{f}\right)} \frac{M_{P l}^{2}}{M^{2}} \approx 1.7 \times 10^{4}$

after dropping the small SM contribution in (24). This relation puts constraints on $M$ and $\Lambda$, depending on how crowded the NP is.

For a Planckian UV cutoff $\Lambda \approx M_{P l}$, the Planck scale in (23) requires $n_{b}-n_{f} \approx 1300$, and this leads to $M / c_{3 / 2} \approx$ $6.3 \times 10^{13} \mathrm{GeV}$. This heavy $\psi_{\mu}$, weighing not far from the see-saw and axion scales, acts as an enabler of Higgs inflation. (Of course, all this makes sense if the $\psi_{\mu}$ contribution in (20) is neutralized by similar contributions from the righthanded neutrinos $v_{R}^{k}$ to alleviate the little hierarchy problem.)

For an intermediate UV cutoff $\Lambda \ll M_{P l}, n_{b}-n_{f}$ can be large enough to bring $M$ down to lower scales. In fact, $M$ gets lowered to $M \sim \mathrm{TeV}$ for $n_{b}-n_{f} \simeq 10^{24}$, and this sets the UV cutoff $\Lambda \sim 3 \mathrm{TeV}$. This highly crowded NP illustrates how small $M$ and $\Lambda$ can be. Less crowded NP sectors lead to intermediate-scale $M$ and $\Lambda$.

It follows therefore that it is possible to realize Higgs inflation through the Higgs-curvature coupling (corresponding to quartic UV-dependence the $\psi_{\mu}$ induces on the Higgs mass). It turns out that Higgs inflation is decided by how heavy $\psi_{\mu}$ is and how crowded the NP is. It is interesting that the $\psi_{\mu}$ hid- den in the SM spectrum enables successful Higgs inflation if gravity is incorporated into the SM as in [5-7].

\section{Spin-3/2 field as dark matter}

Dark matter (DM), forming one-fourth of the matter in the Universe, must be electrically neutral and long-lived. The negative searches [42-44] so far have added one more feature: The DM must have exceedingly suppressed interactions with the SM matter. It is not hard to see that the spin-3/2 fermion $\psi_{\mu}$ possesses all these properties. Indeed, the constraint (4) ensures that scattering processes in which $\psi_{\mu}$ is on its mass shell must all be forbidden simply because its interaction in (6) involves the vertex factor $c_{3 / 2} \gamma^{\mu}$. This means that decays of $\psi_{\mu}$ as in Fig. 1 as well as its co-annihilations with the self- and other SM fields are all forbidden. Its density therefore does not change with time, and the observed DM relic density [45] must be its primordial density, which is determined by the short-distance physics the $\psi_{\mu}$ descends from. It is not possible to calculate the relic density without knowing the short-distance physics. Its mass and couplings, on the other hand, can be probed via the known SMscatterings as studied in Sect. 3 above. In consequence, the $\psi_{\mu}$, as an inherently off-shell fermion hidden in the SM spectrum, possesses all the features required of a DM candidate.

Of course, the $\psi_{\mu}$ is not the only DM candidate in the setup. The crowded NP sector, needed to incorporate gravity in a way solving the hierarchy problem (see Sect. 4 above), involves various fields which do not interact with the SM matter. They are viable candidates for non-interacting DM as well as dark energy (see the detailed analysis in [7]). The noninteracting NP fields can therefore contribute to the total DM distribution in the Universe. It will, of course, not be possible to search for them directly or indirectly. In fact, they do not have to come to equilibrium with the SM matter.

Interestingly, both $\psi_{\mu}$ and the secluded fields in the NP act as extra fields hidden in the SM spectrum. Unlike the $\psi_{\mu}$, which reveal itself virtually, the NP singlets remain completely intact. The main implication is that, in DM phenomenology, one must keep in mind that there can exist an unobservable, undetectable component of the DM [7].

\section{Conclusion and outlook}

In this work we have studied a massive spin-3/2 particle $\psi_{\mu}$ obeying the constraint (4) and interacting with the SM via (6). It hides in the SM spectrum as an inherently off-shell field. We first discussed its collider signatures by studying $v_{L} h \rightarrow$ $v_{L} h$ and $e^{-} e^{+} \rightarrow W^{+} W^{-}$scatterings in detail in Sect. 3. Following this, we turned to its loop effects and determined how it contributes to the big and little hierarchy problems in 
the SM. Resolving the former by appropriately incorporating gravity, we show that the Higgs field can inflate the Universe. Finally, we show that $\psi_{\mu}$ is a viable DM candidate, which can be indirectly probed via the scattering processes we have analyzed.

The material presented in this work can be extended in various ways. A partial list would include:

- Determining under what conditions right-handed neutrinos can lift the constraints on $\psi_{\mu}$ from the neutrino masses.

- Improving the analyses of $v_{L} h \rightarrow v_{L} h$ and $e^{-} e^{+} \rightarrow$ $W^{+} W^{-}$scatterings by including loop contributions.

- Simulating $e^{-} e^{+} \rightarrow W^{+} W^{-}$at the ILC by taking into account planned detector acceptances and collider energies.

- Performing a feasibility study of the proposed neutrinoHiggs collider associated with $v_{L} h \rightarrow v_{L} h$ scattering.

- Exploring UV-naturalness by including right-handed neutrinos, and determining under what conditions the little hierarchy problem is softened.

- Including effects of the right-handed neutrinos into Higgs inflation, and determining appropriate parameter space.

- Giving an in-depth analysis of the dark matter and dark energy by taking into account the spin-3/2 field, righthanded neutrinos and the secluded NP fields.

- Studying constraints on the masses of NP fields from nucleosynthesis and other processes in the early Universe.

We will continue to study the spin-3/2 hidden field starting with some of these points.

Acknowledgements This work is supported in part by the TUBITAK Grant 115F212. We thank the conscientious referee for enlightening comments and suggestions.

Open Access This article is distributed under the terms of the Creative Commons Attribution 4.0 International License (http://creativecomm ons.org/licenses/by/4.0/), which permits unrestricted use, distribution, and reproduction in any medium, provided you give appropriate credit to the original author(s) and the source, provide a link to the Creative Commons license, and indicate if changes were made. Funded by SCOAP ${ }^{3}$.

\section{References}

1. G. Aad et al., [ATLAS and CMS Collaborations], Combined Measurement of the Higgs Boson Mass in $p p$ collisions at $\sqrt{s}=7$ and $8 \mathrm{TeV}$ with the ATLAS and CMS experiments. Phys. Rev. Lett. 114, 191803 (2015). arXiv:1503.07589 [hep-ex]

2. W. Rarita, J. Schwinger, On a theory of particles with half-integral spin. Phys. Rev. 60, 61 (1941)

3. V. Pascalutsa, Correspondence of consistent and inconsistent spin$3 / 2$ couplings via the equivalence theorem. Phys. Lett. B 503, 85 (2001). arXiv:hep-ph/0008026
4. T. Pilling, Symmetry of massive Rarita-Schwinger fields. Int. J. Mod. Phys. A 20, 2715 (2005). arXiv:hep-th/0404131

5. D.A. Demir, A mechanism of ultraviolet naturalness. arXiv: 1510.05570 [hep-ph]

6. D.A. Demir, Curvature-Restored gauge invariance and ultraviolet naturalness. Adv. High Energy Phys. 2016, 6727805 (2016). arXiv:1605.00377 [hep-ph]

7. D. Demir, Naturalizing gravity of the quantum fields, and the hierarchy problem. arXiv:1703.05733 [hep-ph]

8. K.S. Babu, E. Ma, J.W.F. Valle, Underlying A(4) symmetry for the neutrino mass matrix and the quark mixing matrix. Phys. Lett. B 552, 207 (2003). arXiv:hep-ph/0206292

9. W. Grimus, L. Lavoura, A nonstandard CP transformation leading to maximal atmospheric neutrino mixing. Phys. Lett. B 579, 113 (2004). arXiv:hep-ph/0305309

10. E. Ma, Neutrino theory: mass, interactions, connections. PoS CORFU 2015, 009 (2016)

11. O. Sargin, Symmetries of the hidden spin-3/2 field and right-handed neutrinos, and implications for neutrino masses. Work in progress (2017)

12. M. Ruan, Higgs measurement at $e^{+} e^{-}$circular colliders. Nucl. Part. Phys. Proc. 273-275, 857 (2016). arXiv:1411.5606 [hep-ex]

13. M. Bicer et al., [TLEP Design Study Working Group Collaboration], First look at the physics case of TLEP. JHEP 1401, 164 (2014). arXiv:1308.6176 [hep-ex]

14. D. d'Enterria, Physics at the FCC-ee. arXiv:1602.05043 [hep-ex]

15. H. Baer et al., The international linear collider technical design report - volume 2: physics. arXiv:1306.6352 [hep-ph]

16. G. Moortgat-Pick et al., Physics at the e+ e- linear collider. Eur. Phys. J. C 75(8), 371 (2015). arXiv:1504.01726 [hep-ph]

17. K. Fujii et al., Physics case for the international linear collider. arXiv:1506.05992 [hep-ex]

18. S. Choubey et al. [IDS-NF Collaboration], International design study for the neutrino factory, interim design report. arXiv:1112.2853 [hep-ex]

19. M. Bonesini, Perspectives for Muon colliders and neutrino factories. Frascati Phys. Ser. 61, 11 (2016). arXiv:1606.00765 [physics.acc-ph]

20. D.M. Kaplan [MAP and MICE Collaborations], Muon colliders and neutrino factories. EPJ Web Conf .95, 03019 (2015). arXiv: 1412.3487 [physics.acc-ph]

21. J.M. Cornwall, D.N. Levin, G. Tiktopoulos, Derivation of gauge invariance from high-energy unitarity bounds on the s matrix. Phys. Rev. D 10 (1974) 1145 Erratum: [Phys. Rev. D 11 (1975) 972]

22. M.S. Chanowitz, M.K. Gaillard, The TeV physics of strongly interacting W's and Z's. Nucl. Phys. B 261, 379 (1985)

23. M.E. Peskin, D.V. Schroeder, An introduction to quantum field theory

24. M.J.G. Veltman, The infrared-ultraviolet connection. Acta Phys. Polon. B 12, 437 (1981)

25. Z.H. Yu, J.M. Zheng, X.J. Bi, Z. Li, D.X. Yao, H.H. Zhang, Constraining the interaction strength between dark matter and visible matter: II. Scalar, vector and spin-3/2 dark matter. Nucl. Phys. B 860, 115 (2012). arXiv:1112.6052 [hep-ph]

26. R. Ding, Y. Liao, Spin $3 / 2$ particle as a dark matter candidate: an effective field theory approach. JHEP 1204, 054 (2012). arXiv:1201.0506 [hep-ph]

27. R. Ding, Y. Liao, J.Y. Liu, K. Wang, Comprehensive constraints on a spin-3/2 singlet particle as a dark matter candidate. JCAP 1305, 028 (2013). arXiv:1302.4034 [hep-ph]

28. K.G. Savvidy, J.D. Vergados, Direct dark matter detection: a spin 3/2 WIMP candidate. Phys. Rev. D 87(7), 075013 (2013). arXiv:1211.3214 [hep-ph]

29. S. Dutta, A. Goyal, S. Kumar, Anomalous X-ray galactic signal from $7.1 \mathrm{keV}$ spin-3/2 dark matter decay. JCAP 1602(02), 016 (2016). arXiv:1509.02105 [hep-ph] 
30. M.O. Khojali, A. Goyal, M. Kumar, A.S. Cornell, Minimal spin3/2 dark matter in a simple $s$-channel model. arXiv:1608.08958 [hep-ph]

31. M. Capdequi Peyranere, J.C. Montero, G. Moultaka, Is natural fine tuning feasible in the standard model? Phys. Lett. B 260, 138 (1991)

32. A.A. Andrianov, R. Rodenberg, N.V. Romanenko, Fine tuning in one Higgs and two Higgs standard model. Nuovo Cim. A 108, 577 (1995). arXiv:hep-ph/9408301

33. F. Bazzocchi, M. Fabbrichesi, P. Ullio, Just so Higgs boson. Phys. Rev. D 75, 056004 (2007). arXiv:hep-ph/0612280

34. C.N. Karahan, B. Korutlu, Effects of a real singlet scalar on Veltman condition. Phys. Lett. B 732, 320 (2014). arXiv:1404.0175 [hep$\mathrm{ph}]$

35. D.A. Demir, C.N. Karahan, B. Korutlu, Higgsed stueckelberg vector and higgs quadratic divergence. Phys. Lett. B 740, 46 (2015). arXiv:1409.1033 [hep-ph]

36. D.A. Demir, Effects of curvature-higgs coupling on electroweak fine-tuning. Phys. Lett. B 733, 237 (2014) arXiv:1405.0300 [hep$\mathrm{ph}]$

37. B. Korutlu, Softly fine-tuned standard model and the scale of inflation. Mod. Phys. Lett. A 30(34), 1550179 (2015). arXiv:1510.08606 [hep-ph]
38. D.A. Demir, Vacuum energy as the origin of the gravitational constant. Found. Phys. 39, 1407 (2009). arXiv:0910.2730 [hep-th]

39. D.A. Demir, Stress-energy connection and cosmological constant problem. Phys. Lett. B 701, 496 (2011). arXiv:1102.2276 [hep-th]

40. F.L. Bezrukov, M. Shaposhnikov, The standard model higgs boson as the inflaton. Phys. Lett. B 659, 703 (2008). arXiv:0710.3755 [hep-th]

41. F. Bezrukov, A. Magnin, M. Shaposhnikov, S. Sibiryakov, Higgs inflation: consistency and generalisations. JHEP 1101, 016 (2011). arXiv: 1008.5157 [hep-ph]

42. M. Klasen, M. Pohl, G. Sigl, Indirect and direct search for dark matter. Prog. Part. Nucl. Phys. 85, 1 (2015). arXiv:1507.03800 [hep-ph]

43. F. Kahlhoefer, Review of LHC dark matter searches. Int. J. Mod. Phys. A 32(13), 1730006 (2017). arXiv: 1702.02430 [hep-ph]

44. L. Roszkowski, E.M. Sessolo, S. Trojanowski, WIMP dark matter candidates and searches-current issues and future prospects. arXiv:1707.06277 [hep-ph]

45. P.A.R. Ade et al., [Planck Collaboration], Planck 2015 results. XIII. Cosmological parameters. Astron. Astrophys. 594, A13 (2016). arXiv:1502.01589 [astro-ph.CO] 